\title{
Mapping Crisp Structural Semantic Similarity Measures to Fuzzy Context: A Generic Approach
}

\author{
Hoda Safaeipour $^{1}$, M. H. Fazel Zarandi ${ }^{1}, \underline{\text { Susan Bastani }}^{2}$ \\ ${ }^{1}$ Department of Industrial Engineering, Amirkabir University of Technology, 424 Hafez Ave, P.O. Box 15875-4413, \\ Tehran, Iran. \\ ${ }^{2}$ Faculty of Social Sciences and Economics, Department of Sociology, Alzahra University, Tehran, Iran.
}

\section{Abstract}

Ontology-based similarity measures have received much importance in recent years. In many realworld cases, the domain considered in the ontological similarity assessment consists of uncertainty or incomplete information. Such vagueness has led to the successful implementation of fuzzy ontology (FO)-based similarity measures. Despite various applications of FO-based similarity measures, limited methods have so far been proposed for this purpose. Accordingly, this paper presents a generic model for semantic similarity assessment based on a fuzzy ontology. The proposed approach relies on the broad literature of Crisp Ontology-based Structural Semantic Similarity Measures (COSSSM). It provides an approach for mapping CO-SSSMs to fuzzy context. Consequently, the proposed generic model can be applied to various COSSSMs to develop their corresponding FO-SSSMs. In this regard, as an empirical investigation, four of the common CO-SSSMs were selected, their equivalent FO-SSSMs were developed by means of the proposed approach, and the accuracy of their similarity assessment was compared with each other. The results show the power of FO-SSSMs in describing the relations between concepts and their superiority over CO-SSSMs.

Keywords: Similarity measure, Fuzzy ontology, Semantic reasoning. Generic model. 